\title{
ARTIGOS
}

\section{LA SOCIOLOGÍA DEL SISTEMA DE ENSEÑANZA DE BOURDIEU: REFLEXIONES DESDE AMÉRICA LATINA}

MERCEDES MOLINA GALARZA

\section{RESUMEN}

En este trabajo abordamos la teoría del sistema de enseñanza de Pierre Bourdieu, concebida en sus últimas obras como campo escolar. Enfocamos la relación entre estrategias escolares -en el marco de estrategias familiares de reproducción social- y la "elección de los elegidos" que realiza la escuela. Revisamos algunas críticas y debates relativos a este marco conceptual, considerando la cuestión de la importación teórica en América Latina; la presencia de explicaciones funcionalistas y los límites heurísticos de algunos conceptos claves, como campo y habitus. También recuperamos aspectos centrales de la noción de campos periféricos. Por último, estimamos las posibilidades que ofrece este arsenal teórico para contribuir a una necesaria democratización de los procesos y escenarios escolares.

SOCIOLOGÍA DE LA EDUCACIÓN • PIERRE BOURDIEU • DESIGUALDAD SOCIAL • SISTEMA ESCOLAR

\section{BOURDIEU'S SOCIOLOGY OF EDUCATION: CONSIDERATIONS FROM LATIN AMERICA}

\section{ABSTRACT}

In this paper we address Pierre Bourdieu's theory of education, conceived in his later works as an educational field theory. We analyze the relationship between school strategies -under certain family strategies of social reproduction-and the process of "selecting the elect" conducted by the school. We review some criticisms and discussions related to this conceptual framework, considering the issue of theory importation in Latin America; the presence of functionalist explanations and the heuristic limits of some key concepts, such as field and habitus. We also consider some central aspects of the concept of peripheral fields. Finally, we estimate the possibilities offered by this theoretical arsenal to contribute to the necessary democratization of school processes and settings.

SOCIOLOGY OF EDUCATION • PIERRE BOURDIEU • SOCIAL INEQUALITY • SCHOOL SYSTEM 


\section{LA SOCIOLOGIE DU SYSTĖME D’ENSEIGNEMENT DE BOURDIEU: RÉFLÉXIONS À PARTIR DE L'AMÉRIQUE LATINE \\ RÉSUMÉ}

Ce travail abordera la théorie du système d'enseignement de Pierre Bourdieu, conçue comme champ scolaire dans ses derniers travaux. L'accent sera mis sur la relation entre les stratégies scolaires - dans le cadre des stratégies familiales de reproduction sociale - et le "choix des élus", réalisé par l'école. Une révision de critiques et débats relatifs à ce cadre conceptuel sera entreprise, à partir de la question de l'importation théorique en Amérique Latine, de la présence d'explications fonctionnalistes et des limites heuristiques de certains concepts clés, comme ceux de champ et d' habitus. Les principaux aspects de la notion de champs périphériques seront aussi abordés. La conclusion se portera sur les possibles contributions de cet arsenal théorique à la nécessaire démocratisation des processus et scénarios scolaires.

SOCIOLOGIE DE L'ÉDUCATION • PIERRE BOURDIEU • INÉGALITÉ SOCIALE • SYSTĖME SCOLAIRE

\section{A SOCIOLOGIA DO SISTEMA DE ENSINO DE BOURDIEU: REFLEXÕES A PARTIR DA AMÉRICA LATINA \\ RESUMO}

Neste trabalho abordamos a teoria do sistema de ensino de Pierre Bourdieu, concebida em suas últimas obras como campo escolar. Focamos a relação entre estratégias escolares - no âmbito de estratégias familiares de reprodução social - e a "escolha dos escolhidos" realizada pela escola. Revemos algumas críticas e debates relativos a esse marco conceitual, considerando a questão da importação teórica na América Latina; a presença de explicações funcionalistas e os limites heurísticos de alguns conceitos chaves, como campo e habitus. Também recuperamos aspectos centrais da noção de campos periféricos. Por último, estimamos as possibilidades que esse arsenal teórico oferece a fim de contribuir para uma necessária democratização dos processos e cenários escolares. 
Este trabajo fue financiado parcialmente con fondos del PICT 2011-1736 Subjetividades, conflictos y estrategias en centros educativos de nive secundario para adultos/as en Mendoza. Una mirada sociológica (Agencia Nacional de Promoción Científica y

Tecnológica) y, parcialmente con fondos del Proyecto

06/F351: Representaciones sociales y experiencia escolar en la educación de jóvenes y adultos. Un estudio de casos en Gran Mendoza (Secretaría de Ciencia, Técnica y Posgrado, UNCuyo, 2013) República Argentina.

Lo anterior no quita la existencia de grandes movimientos sociales y significativas producciones teóricas que se constituían en críticos y detractores del sistema capitalista, como modo de organización de la producción y la vida social.

UCHO SE HA ESCRITO Y DEBATIDO ACERCA DE QUE, TRAS LA CAÍDA DE LOS SOCIALISMOS reales y el fin de la guerra fría, el capitalismo de fines del siglo pasado pareció haber llegado a un punto de máxima hegemonía. No sólo había visto desmoronarse al sistema contra el cual luchó a lo largo de casi un siglo, sino que gozaba, como nunca antes quizás, de un extendido consenso. ${ }^{1}$ Diez años antes de la caída del muro de Berlín, Jean-François Lyotard vaticinaba en 1979 este cambio de época al afirmar la llegada de la posmodernidad y el fin de los grandes relatos. Era también el fin de la utopía socialista y las teorías emancipatorias contrarias al capitalismo.

La obra de Pierre Bourdieu no es, por cierto, ni una ni las otras. Lejos está de ofrecer un legado esperanzado o enfocado hacia la utopía de una sociedad mucho mejor que la que conocemos. Sin embargo, la vigencia de su arsenal conceptual desafía los vaticinios teórico-políticos de Lyotard. Su potencial radica, quizás, en su ubicuidad: se trata de un conjunto de herramientas que habilitan el análisis de ámbitos variados de la vida social, en la actualidad o a lo largo de la historia. Claro, al hacer visibles relaciones de fuerza, jerarquías sociales y desigualdades, no puede menos que ser una teoría profundamente crítica y desestabilizadora de los discursos legitimatorios de las sociedades capitalistas contemporáneas.

Se trata de una crítica surgida del seno mismo del poder: Bourdieu escribe desde París, Francia; un país central, con una academia mundialmente reconocida. Pero además, es un catedrático varón, 
heterosexual, blanco y sus trabajos son una impugnación a las formas de organización de la vida social basadas en la opresión capitalista, el patriarcado y el racismo. Muchos otros esfuerzos teóricos han apuntado contra estas formas de dominación. Sin embargo, la obra de Bourdieu tiene la virtud de reunir todos sus aportes en un solo y vasto conjunto de herramientas teórico-metodológicas. Además, como intentaremos fundamentar en las páginas siguientes, tiene mucho para aportar a la labor sociológica que busca dar cuenta de las relaciones sociales del siglo XXI en sociedades periféricas como las latinoamericanas.

Esta es la primera de las razones por las cuales consideramos necesario el estudio sistemático de su legado teórico. Pero además, hay otras. Sus obras han sido ampliamente citadas y criticadas aunque, a nuestro juicio, insuficientemente leídas o profundizadas. En América Latina, es frecuente el uso de conceptos y definiciones aisladas, sin atender al significado que adquieren dentro del universo conceptual del que forman parte, solidario con una perspectiva crítica y una vocación política de denuncia del statu quo.

En el presente trabajo, proponemos una lectura de la sociología del sistema de enseñanza del autor que tiene como punto de partida la consideración de las estrategias escolares como parte de las estrategias de reproducción social de las familias a las que pertenecen. Está claro que la trayectoria del estudiante individual que se hace observable desde la escuela no tiene, desde esta óptica, nada de individual. Es resultado de un entramado más amplio gracias al cual la vida familiar se desenvuelve. Las familias hacen sus “apuestas” educativas para el futuro de los hijos, al tiempo que satisfacen las necesidades del presente y buscan mantener o mejorar las posiciones sociales ocupadas. El resultado de las estrategias educativas (léase, el éxito o fracaso escolar) se explica por medio de la metáfora de la "elección de los elegidos" que realiza la escuela y que da cuenta de los buenos rendimientos académicos alcanzados por estudiantes de clases socialmente favorecidas, así como del rendimiento deficitario y la corta duración de los estudios de aquellos de origen popular.

A continuación, hemos tomado en cuenta algunas perspectivas críticas en relación a la obra de Bourdieu. Analizamos, entre otras cuestiones, las posibilidades de la importación teórica a los contextos sociales latinoamericanos; las limitaciones de la teoría del sistema de enseñanza, toda vez que recae en explicaciones funcionalistas de la vida en las escuelas, al igual que los límites heurísticos de algunos de sus conceptos claves, como el concepto de campo y el de habitus. También se toman en consideración aspectos que resultan fundamentales para el empleo de la noción de campos periféricos.

Finalmente, hemos revisado algunas de las conclusiones a las que arriba la sociología de la educación bourdieana, para considerar si 
la escuela está ineludiblemente comprometida con la reproducción de las inequidades sociales o si es posible imaginar el modo en que podría colaborar en la construcción de una sociedad más democrática.

\section{ESTRATEGIAS FAMILIARES Y ESTRATEGIAS ESCOLARES}

Para comprender qué sucede al interior del sistema de enseñanza, es necesario primero comprender que las estrategias escolares forman parte de un sistema más amplio, en el que se incluyen todas aquellas prácticas destinadas a la producción y reproducción de la vida de los individuos y las familias, que el autor ha denominado estrategias de reproducción social. Se trata de un

\section{[...] conjunto de prácticas fenomenalmente muy diferentes, por medio de las cuales los individuos y las familias tienden, de manera consciente o inconsciente, a conservar o aumentar su patrimonio, y correlativamente a mantener o mejorar su posición en la estructura de las relaciones de clase. (BOURDIEU, 2006, p. 122)}

La finalidad de este sistema de estrategias es la producción y reproducción de la vida de la unidad familiar y de cada uno de sus miembros, optimizando la utilización de los recursos disponibles y garantizando su transmisión a las nuevas generaciones. En tal sentido, se trata de prácticas

\section{[...] por las cuales la familia tiende a reproducirse biológicamente y sobre todo socialmente, es decir, a reproducir las propiedades que le permitan mantener su posición, su rango en el universo social considerado. (BOURDIEU, 1988, p. 75)}

Dentro del amplio abanico de estrategias de reproducción social, que involucra a la familia y a cada uno de sus integrantes e incluye estrategias matrimoniales, de fecundidad, económicas, de preservación del capital biológico, entre otras, encontramos las estrategias educativas. Con ellas, las familias procuran garantizar la transmisión de capital simbólico a los miembros de las nuevas generaciones e intentan prepararlos para mantener o mejorar su posición en el espacio social. Además, las estrategias de reproducción social constituyen un sistema y están cronológicamente articuladas. Esto quiere decir que las prácticas o estrategias implementadas en un campo afectan o delimitan las posibilidades futuras en otros. También es relevante señalar que, para Bourdieu, las estrategias de reproducción social "contribuyen 
en la práctica a la reproducción del sistema completo de diferencias constitutivas del orden social” (2011, p. 37).

Las familias realizan, a lo largo de extensos períodos de tiempo, una inversión a futuro que consiste en mandar a los/as hijos/as a la escuela. ${ }^{2}$ Es aquí donde hallamos el punto de encuentro entre las estrategias familiares y el sistema de enseñanza. ¿Qué es lo que sucede específicamente dentro de la institución escolar, según Pierre Bourdieu? A través de complejos mecanismos, la escuela contribuye a reproducir la distribución del capital cultural, y colabora con ello a la reproducción de la estructura del espacio social. La lógica académica, aparentemente meritocrática, tiende a otorgar a los estudiantes un capital escolar bajo la forma de títulos (credenciales educativas) en los cuales queda reconocido y legitimado el capital cultural detentado por la familia de origen y transmitido a los/as hijos/as a lo largo de la educación familiar, para el caso de las familias posicionadas en los peldaños más altos de la estructura social. La escuela funciona entonces "seleccionando" a quienes ya detentan mayores porciones de capital cultural heredado de sus familias de origen, pero ¿cómo lo hace?

El ya clásico texto La reproducción. Elementos para una teoría del sistema de enseñanza (BOURDIEU; PASSERON, 1970/1996) concibe taxativamente a toda acción pedagógica como violencia simbólica en la medida que está destinada a imponer, por medio de un poder arbitrario (la autoridad escolar) una arbitrariedad cultural (la cultura de la clase dominante). De este modo, la escuela se reserva el monopolio de la violencia simbólica legítima, aun cuando se presente a sí misma y a sus educadores como los defensores de pedagogías libres, naturales o no represivas. Por supuesto, es fundamental que los miembros de esa formación social, particularmente los estudiantes y sus familias, otorguen legitimidad a las acciones escolares, reconociendo tanto la autoridad de los maestros como la validez de los contenidos que la escuela transmite. También los mecanismos del mercado consolidan la acción del sistema escolar, dado que sancionan positivamente una arbitrariedad cultural (ciertos contenidos de cultura) a los que consideran legítimos y les confieren mayor valor en los intercambios mercantiles, mientras otorgan un valor menor a los elementos propios de las culturas dominadas.

La arbitrariedad de los contenidos escolares no aparece nunca en su completa verdad, así como tampoco se presenta a sí mismo como violento el poder arbitrario de imposición del que gozan los maestros. La inculcación es más eficaz en la medida en que los estudiantes e incluso los docentes no sean conscientes de esto, es decir, en tanto haya un desconocimiento de lo que Bourdieu y Passeron denominan la "verdad objetiva” de la acción pedagógica, concebida en cambio como autoridad necesaria y natural. todos los niveles del sistema, desde la educación básica hasta el nivel superior. 
Un pasaje memorable de La reproducción, cuya retórica parece hoy seguramente exagerada, expresaba:

En la medida en que el éxito de toda acción pedagógica es función del grado en el que los receptores reconocen la autoridad pedagógica de la instancia pedagógica y del grado en que dominan el código cultural de la comunicación pedagógica, el éxito de una determinada acción pedagógica en una formación social determinada está en función del sistema de relaciones entre la arbitrariedad cultural que impone esta acción pedagógica, la arbitrariedad cultural dominante en la formación social considerada y la arbitrariedad cultural inculcada por la primera educación en los grupos o clases de donde proceden los que sufren esta acción pedagógica. (BOURDIEU; PASSERON, 1970/1996, p. 70)

El éxito escolar (es decir, el hecho de aprobar los exámenes y lograr la promoción en los sucesivos niveles de escolarización) depende del grado en que los estudiantes dominan el "código cultural” necesario para descifrar y apropiarse de la arbitrariedad cultural que se impone en la escuela. Así, los alumnos/as cuya socialización familiar les inculcó unos habitus y una cultura que se corresponden con la arbitrariedad cultural dominante (que es la que la escuela transmite en un momento dado, en una formación social determinada) tienen mayores probabilidades de avanzar exitosamente en sus estudios, puesto que dominan el código (los rudimentos, las bases) de la cultura considerada legítima. Por el contrario, los estudiantes oriundos de las clases menos favorecidas sólo podrán avanzar satisfactoriamente en su escolarización en la medida en que dejen atrás sus culturas de origen y adquieran, a costa de un alto trabajo de aculturación (que pocos pueden lograr), los habitus y los contenidos propios de la cultura dominante.

La importancia del habitus, y no solamente de los contenidos culturales trasmitidos, se pone de manifiesto en la siguiente afirmación:

El grado de productividad específica de cualquier trabajo pedagógico [...] está en función de la distancia que separa el habitus que tiende a inculcar (o sea, la arbitrariedad cultural impuesta) del habitus inculcado por los trabajos pedagógicos anteriores. (BOURDIEU; PASSERON, 1970/1996, p. 83)

Aunque los estudiantes puedan incorporar nuevos contenidos de manera consciente, opera muy fuertemente aquella disposición inconsciente para el trabajo intelectual que está en el origen de las estrategias escolares y genera, cotidianamente, todas aquellas prácticas orientadas a la tarea de estudiar. Además, Bourdieu ha destacado 
la necesidad de tener en cuenta lo que llama tecnología del trabajo intelectual, que constituye un saber-hacer que no se encuentra igualmente distribuido entre la población escolar, como se lee en el siguiente fragmento:

[...] hay cosas de las cuales todo el mundo hace como si todos
las poseyeran, mientras que solamente algunos las dominan;
por ejemplo, el hecho de saber tomar notas, el hecho de saber
hacer una ficha, utilizar un diccionario, el uso de las abreviaturas,
la retórica de la comunicación, la organización de un fichero, la
creación de un índice, la utilización de un fichero descriptivo de
un banco de datos, de una biblioteca, el uso de instrumentos
informáticos, la lectura de cuadros estadísticos y de gráficas. La
tecnología del trabajo intelectual no solamente no se nos enseña,
sino que es menospreciada. (BOURDIEU, 2005, p. 170)

A menudo, los estudiantes desconocen que necesitan de estas técnicas para el trabajo académico. Por otra parte, no se trata de conocimientos o disposiciones que se puedan adquirir de la noche a la mañana. Sólo a costa de grandes esfuerzos, los/as alumnos/as de origen popular pueden recorrer con éxito estudios -sobre todo en el nivel superior- para los que no estaban inicialmente preparados. Así, desde el punto de vista del autor, estos estudiantes están obligados a realizar un trabajo muchísimo más duro -dejando atrás su cultura de origen y asimilando la cultura legítima, pero también, los habitus asociados a ella- para obtener, con suerte, los mismos resultados que el resto de sus compañeros, herederos de capital cultural, habitus y posiciones más elevadas en el espacio social.

\section{LA "ELECCIÓN DE LOS ELEGIDOS" ¿UNA PERSPECTIVA VIGENTE?}

La teoría sociológica de Pierre Bourdieu no flota en el aire, sino que sus proposiciones descansan sobre largas series de observaciones y datos empíricos, obtenidos a partir de fuentes diversas. Desde el análisis de datos censales y encuestas estadísticamente representativas, hasta entrevistas cualitativas, observación en terreno y análisis de casos, sus investigaciones han empleado métodos y técnicas de corte tanto cuantitativo como cualitativo. No constituye ninguna obviedad detenernos en esto, debido a que sus trabajos suelen ser criticados por sus conclusiones, sin prestar suficiente atención al material empírico que le sirve de sustento. Una crítica bien fundada no debería menos que tomar en consideración la casuística sobre la cual se ha basado, sea para objetar el modo en que los datos fueron construidos (crítica metodológica 
y/o epistemológica), sea para ofrecer otras interpretaciones diferentes acerca del mismo material empírico (crítica teórica).

En 1964, salía a la luz una obra que se constituiría en un clásico de la sociología de la educación crítica, y antesala de La reproducción. Se trata de Los herederos. Los estudiantes y la cultura (BOURDIEU; PASSERON, 1964/2006). En ella, los autores evalúan una larga serie de materiales estadísticos, de fuentes primarias (elaboración propia) y secundarias (resultados censales) en donde se revelan grandes tendencias y regularidades de la vida estudiantil en Francia, durante las primeras seis décadas del siglo XX.

Allí se ponen de manifiesto procesos como la incorporación progresiva de estudiantes y la masificación de la enseñanza lo largo del período señalado, o la importancia del origen social (operacionalizado en la categoría “condición socio profesional” del padre ${ }^{3}$ ) en el desempeño universitario de los estudiantes. Las "elecciones" vocacionales diferenciales según el sexo también se tornan visibles, mostrando la "preferencia” de las mujeres por carreras universitarias en las que continúan desempeñando los roles que socialmente les han sido atribuidos -como profesorados, humanidades y artes- mientras los varones se vuelcan hacia disciplinas tradicionales como “ciencias”, derecho y medicina.

Los resultados numéricos podrían parecer, en sí mismos, poco significativos, pues ha transcurrido ya más de medio siglo y nuestra mirada se sitúa en un escenario social muy alejado del centro francés: la Argentina de la segunda década del siglo XXI. Sin embargo, esto no es más que una apreciación apresurada, pues si los datos parecen desactualizados o se refieren a latitudes muy alejadas a la nuestra, lo cierto es que las generalizaciones teóricas a las que han dado lugar resultan atractivas o, como mínimo, sugerentes para pensar los procesos que se suceden en nuestras escuelas hoy. En ese texto fundante de una

En Argentina Susana Torrado realiza una operacionalización similar de la clase social para sus investigaciones estadísticas, considerando como indicador la categoría socioocupacional del jefe de hogar (2003). En ese mismo libro, la autora emplea e concepto de estrategias familiares de vida. expresamente emparentado con el concepto bourdieano de estrategias de reproducción social. muchos aspectos de la concepción bourdieana de la inteligencia como "ideología del don" y profundiza los alcances de la crítica es la de Kaplan (2008) nueva sociología de la educación -la perspectiva crítica- las estadísticas muestran cómo lo que se presenta como el resultado de la libre elección individual, o del esfuerzo personal y el mérito de la inteligencia, ${ }^{4}$ es en realidad el producto de un proceso de selección escolar donde se lleva a cabo la "elección de los elegidos", aquellos/as a los que el sistema social necesita ungir como herederos de la cultura legítima, en función del sexo y el origen social.

En esta novedosa -para su contexto de surgimiento- aproximación al sistema de enseñanza encontramos, sin embargo y como ha señalado Martín Criado (2003, 2010), elementos explicativos funcionalistas. Lo que podrían ser efectos de la acción escolar, se trastocan en ocasiones -como parte de una serie de operaciones retóricas- en funciones de la misma: la escuela contribuye a la reproducción de las relaciones de producción capitalistas y tal es su función. No se trata de un enfoque legitimatorio del accionar escolar y su contribución al mantenimiento del orden 
social, como es el caso del funcionalismo parsoniano. Es, en cambio, una perspectiva que deja en evidencia las profundas inequidades del sistema de enseñanza, recurriendo para ello a la lógica de las "funciones escolares”, especialmente en el texto de La reproducción. Por su parte, en la obra Los herederos también es posible encontrar pasajes que van en esa dirección, en forma si se quiere muy esporádica pero no poco contundente. Así, por ejemplo, se dice que "Entre otras funciones, el sistema educativo debe producir sujetos seleccionados y jerarquizados de una vez para siempre y para toda la vida" (BOURDIEU; PASSERON, 1964/2006, p. 104). Aunque sean contadas a lo largo del texto, este tipo de nociones funcionalistas están presentes y resultan insoslayables.

Dos años después de la aparición de Los Herederos y cuatro años antes de La reproducción, Bourdieu ofrece una primera formulación de la noción de campo en el texto Campo intelectual y proyecto creador, que data del año 1966. Podemos decir que la idea de campo está ya "en proceso de elaboración” en la segunda mitad de la década de los 60. Sin embargo, en La reproducción la noción no aparece; de hecho, no se menciona allí ningún “campo escolar” o "campo académico” y, en cambio, se hace referencia al sistema de enseñanza.

Casi dos décadas después, obras de madurez como Homo academicus (BOURDIEU, 1984/2008) y La nobleza de Estado (BOURDIEU, 1989/2013) incorporan la perspectiva de campo al abordaje de problemáticas educativas. Se trata de una investigación enfocada en la educación universitaria, en el primero de los casos, y su relación con los puestos patronales o gerenciales -en empresas públicas o vinculadas al Estado y en empresas privadas- en el segundo. En ellas, se consideran tanto el campo del profesorado universitario, el campo intelectual y científico, así como sus interrelaciones con el campo del poder. ${ }^{5}$ Más allá de este giro teórico respecto de sus primeras obras sobre el sistema de enseñanza, coincidimos con Martín Criado (2004) en que algunas de las explicaciones funcionalistas siguen presentes y operando en los análisis. Si ello podría traer consigo dificultades y obstáculos para la labor sociológica, es algo de lo que nos ocuparemos en el apartado siguiente.

En términos generales, son muchas las críticas que se pueden hacer al análisis del sistema de enseñanza inaugurado por Bourdieu y Passeron, por su carácter irremediablemente reproductivista y desesperanzado, incluso por su funcionalismo. Pero, sin duda, la pregunta que sigue teniendo vigencia o a la que la sociología de la educación sólo ha podido hallarle respuestas provisorias es cómo lograr que la escuela se transforme en una institución que ofrezca al menos un mínimo de oportunidades a los estudiantes de todos los orígenes sociales incluyendo, particularmente, a los de origen popular. La meritocracia escolar y la igualdad de oportunidades son una falacia, afirmaban Bourdieu y Passeron en 1964. Los procesos de selección 5 Bourdieu define la estructura del campo universitario como "el estado, en un determinado momento del tiempo, de la relación de fuerzas entre los agentes o, más exactamente, entre los poderes que ellos detentan a título personal y sobre todo a través de las instituciones de las que forman parte; la posición ocupada en esta estructura está en el principio de las estrategias que apuntan a transformarla o a conservarla modificando o manteniendo la fuerza relativa de los diferentes poderes o, si se prefiere, las equivalencias establecidas entre las diferentes especies de capital" (1984/2008, p. 171). Uno de los objetivos de Homo academicus es precisamente mostrar cómo esa estructura se transforma a lo largo del tiempo, siendo Mayo del 68 uno de los momentos de crisis y cambio analizados. 
escolar continúan beneficiando a los hijos de las clases socialmente más favorecidas, al tiempo que perjudican a los miembros de los grupos sociales peor posicionados en el espacio social. La escuela del siglo XX no logró revertir esta tendencia, como demuestran las más variadas perspectivas dentro de la sociología de la educación (GIROUX, 2004; TEDESCO, 2012; BAUMAN, 2013; DUBET, 2015). Por ello queda pendiente, en adelante, la búsqueda de nuevas posibilidades para la democratización escolar.

En cuanto a los límites del marco teórico bourdieano, dedicamos el próximo apartado a una aproximación a algunas de las críticas que ha recibido. Las mismas no han sido formuladas exclusivamente con relación al sistema de enseñanza, sino también con respecto al esquema conceptual más amplio que incluye las categorías de campo, habitus y espacio social. Hemos procurado desarrollar las derivaciones que esas críticas más generales tienen para el análisis sociológico de la institución escolar.

\section{“LAGUNAS”, CRÍTICAS Y DEUDAS DE LA SOCIOLOGÍA DE LA EDUCACIÓN BOURDIEANA}

La obra de Pierre Bourdieu ha despertado amores y odios en los más variados ámbitos académicos y latitudes. Sus detractores lo han calificado de economicista, marxista encubierto, weberiano encubierto, estructuralista y funcionalista, entre otros atributos. Ana Teresa Martínez nos dice que desde el seno del campo académico francés se ha hablado de él en términos de "jefe de secta”, de su "rigidez y absolutismo", también de su "fuerte acento positivista". Logró una significativa acumulación de poder científico y editorial aunque quizás poco poder propiamente académico, contradicción que resultó para él difícil de aceptar (MARTINEZ, 2008).

Algunas de las miradas descalificantes provienen de lecturas poco atentas a los reparos y límites de la teoría que el propio autor ha expresado. Otras, en cambio, tienen fundamentos que consideramos deben ser tomados en cuenta. Nos interesa retomar algunas de esas perspectivas; nos abocamos, a continuación, al tratamiento de esos cuestionamientos. Hemos seleccionado aquellos que entendemos son de utilidad para reflexionar sobre posibles usos de la sociología de la educación bourdieana en los campos periféricos de las sociedades latinoamericanas.

\section{SOBRE LAS POSIBILIDADES DE LA IMPORTACIÓN TEÓRICA}

Una crítica difundida señala que las categorías empleadas por Bourdieu para comprender la vida social francesa son quizás útiles para el estudio de sociedades nacionales similares, como las europeas, pero 
no son extrapolables a la heterogeneidad de escenarios y relaciones sociales en América Latina. Néstor García Canclini entiende que en nuestra región no existe una estructura de clases unificada, en tanto "el modo de producción capitalista incluye diversos tipos de producción económica y simbólica” (1990, p. 31). En lo que toca al campo simbólico latinoamericano, éste se halla fragmentado y se caracteriza por una importante diversidad cultural, propia de nuestras sociedades multiétnicas. Así, la relación entre los lugares sociales ocupados por determinados grupos o sectores y su acceso al mercado de bienes simbólicos es más compleja que en las sociedades europeas. En consecuencia,

\begin{abstract}
[...] coexisten capitales culturales diversos: los precolombinos, el colonial español, en algunos la presencia negra y las modalidades contemporáneas de desarrollo capitalista. [...] Necesitamos reformular la concepción de Bourdieu, en muchos sentidos útil para entender el mercado de bienes simbólicos, a fin de incluir los productos culturales nacidos de los sectores populares, las representaciones independientes de sus condiciones de vida y la resemantización que hacen de la cultura dominante de acuerdo con sus intereses. (GARCíA CANCLINI, 1990, p. 31)
\end{abstract}

García Canclini (1990) cuestiona la relación lineal entre lo que podría denominarse cultura de elite y las condiciones materiales de vida de la burguesía, por un lado, o entre la estética popular y las posiciones de clase menos favorecidas, por el otro, indicando que tal dicotomía no es tan fácilmente observable para el caso de lo que denomina culturas híbridas latinoamericanas. De alguna manera, nos alerta acerca de las operaciones de importación teórica acríticas y desatentas a las particularidades de los contextos sociales de destino. Entendemos que se trata de una vigilancia que siempre debe ser puesta en práctica, para cualquier marco categorial elaborado en latitudes diferentes a las nuestras. El propio Bourdieu advirtió acerca de los alcances de sus elaboraciones teóricas, que no trascienden los límites de la Francia del siglo XX para el caso de los estudios del sistema de enseñanza o del campo académico que nos ocupan.

La cuestión del origen multiétnico, el carácter fragmentado y continuamente resemantizado de los significados presentes en nuestras culturas planteado por García Canclini es relevante a la hora de analizar el campo artístico y cultural. ${ }^{6}$ No obstante, puertas adentro de las escuelas latinoamericanas y también en términos históricos, la cultura considerada legítima ha sido siempre la de las clases mejor posicionadas en el espacio social local, constituidas por los descendientes de los conquistadores europeos blancos. La "historia universal" enseñada es García Canclini es relevante, además, porque ha sido retomada y ha contado con gran aceptación a lo largo y ancho de la región, incluso hasta hoy. Véase, entre los muchos abordajes y recepciones de Bourdieu en América Latina, el reciente trabajo de la autora chilena Moraña (2014) donde, una vez más, los argumentos

del autor de Culturas hibridas nuevamente entran en escena. 
la historia que tiene a Europa (y luego a los Estados Unidos, durante el siglo XX) como centro; la lengua oficial es el español o el portugués, la enseñanza de las artes toma como referencia el arte de los países centrales (nuevamente, Europa y Estados Unidos); las clases de economía tienen a los principios del libre mercado como fundamento, por tomar sólo algunos ejemplos. Así, el esquema bourdieano que reconoce y denuncia el predominio de una cultura escolar solidaria con los intereses y cosmovisión de las clases socialmente mejor posicionadas tiene, para nosotros, validez también para el caso latinoamericano, si consideramos que estos grupos sociales han logrado establecer parámetros -siempre móviles, pero no por ello menos eficaces- de distinción de la cultura legítima, reconocida social y mercantilmente como tal. Al hablar de cultura legítima, nos referimos a aquélla definida no sólo por parámetros regionales o continentales, sino en el marco del sistema-mundo.7

Dicho lo anterior, dejamos respondida de alguna manera la pregunta acerca de si puede resultar útil y válido para la sociología de la educación latinoamericana emplear el arsenal teórico del sociólogo francés. A continuación y haciendo un viraje en el análisis, nos detendremos en dos líneas críticas que provienen del seno de la sociología europea. Estas tienen como blanco a la teoría misma, es decir, al conjunto de herramientas conceptuales legadas y sus alcances. Se trata de las lecturas que Enrique Martín Criado y Bernard Lahire han realizado respecto de la obra de Bourdieu. Más puntualmente, Martín Criado concentra la crítica en su sociología del sistema de enseñanza, en tanto Lahire realiza un análisis más generalizado de los trabajos de su coterráneo francés, con señalamientos puntuales referidos al sistema escolar.

\section{SOBRE LOS LÍMITES DE LA EXPLICACIÓN FUNCIONALISTA}

El catedrático español Enrique Martín Criado (2003, 2004, 2010) identifica dos diferentes enfoques explicativos en la sociología de

Yendo mucho más lejos aún, podríamos afirmar que la cultura socialmente tenida como legítima es heredera de la colonialidad del poder, es eurocéntrica y se caracteriza por un racismo epistémico; en la misma lógica, debe tomarse en cuenta la "colonialidad del saber". Al respecto puede consultarse e fructífero análisis de Segato (2015) sobre la vigencia de la perspectiva de la colonialidad del poder. cuyo autor pionero es e peruano Aníbal Quijano. Reconocemos como labor pendiente que deberemos darnos a futuro el análisis de las posibilidades de conjugar elementos de esta perspectiva con elementos de la sociología bourdieana. la educación de Pierre Bourdieu. Uno de ellos -que será el principal blanco de su crítica, como ya adelantamos en el apartado anterior- es el funcionalista, y supone dar cuenta del sistema escolar por la función que cumple, esto es, reproducir el sistema y las desigualdades sociales. El texto en el que este esquema explicativo cobra importancia central es precisamente La reproducción, aunque en Los herederos esta dificultad no estaría presente. En ambos textos se prefiguran tres conceptos que luego serán fundamentales en la explicación sociológica bourdieana: capital cultural, capital lingüístico y habitus. La obra La reproducción retomará esos conceptos de Los herederos, los "sistematizará y formalizará [...] al tiempo que les hará sufrir una transformación fundamental” (MARTIN CRIADO, 2004, p. 73). Siendo no ya sólo su "efecto" sino la función principal del sistema de enseñanza reproducir las desigualdades y legitimarlas, todo ello ocurre de manera velada, aunque formalmente igualitaria. 
En otras palabras, las estrategias y prácticas que generan los distintos grupos de actores a partir de sus habitus son lo que explica que se produzca una reproducción y [al mismo tiempo] lo explicado por el concepto de reproducción. (MARTIN CRIADO, 2004, p. 82)

Esta suerte de explicación tautológica presenta limitaciones e inconsistencias, abordadas en detalle por el autor. Implica, en primer término, el pasaje desde un nivel de análisis a otro diferente: se pasa así desde una explicación referida a dinámicas interrelacionadas y producidas históricamente (los “efectos” de la acción escolar) a otro tipo de explicación que refiere a estructuras -sistema escolar y sistema social- que de hecho se reproducen y tienen, por ende y al mismo tiempo, la "función" de reproducirse. En este último esquema explicativo funcionalista, la investigación histórica de los sistemas escolares estaría en cierto modo obturada, pues se dirigiría a la mera búsqueda de las condiciones de posibilidad para el cumplimiento de sus funciones, dando por supuesto el ajuste perfecto entre sistema educativo y orden social. En la medida en que el orden social cambia -y tal es lo que sucede con la sociedad francesa y su estratificación a medida que avanza el siglo XX, por tomar el mismo caso analizado por Bourdieu y Passeron (1964/2006) qué de él permanece y se reproduce o cuánto de él se transforma son aspectos que quedan fuera del marco explicativo funcionalista, ha destacado Criado. Podemos agregar aquí que precisamente la dificultad para explicar los cambios histórico-sociales es uno de los aspectos que más han sido criticados de las perspectivas sociológicas de raigambre funcionalista. Retomando la crítica de Martín Criado, si la reproducción es sólo una metáfora, no es suficiente entonces como mecanismo explicativo.

Con un método diferente, que se vale plenamente del análisis histórico y de un enfoque teórico que prefiere el concepto de campo al de reproducción, Martín Criado (2010) destaca el carácter productor del campo escolar en el espacio social. Subraya, por ejemplo, el papel de la escuela en la producción de lenguaje y normas lingüísticas, o su papel en la producción cultural. Enfatiza la necesidad de indagar en las mutuas relaciones entre el sistema educativo y los campos de producción científica y cultural, en lugar de establecer a priori que uno reproduce a los otros.

El concepto de campo "es una poderosa herramienta para abordar el sistema escolar escapando a las trampas del pensamiento funcionalista" ha expresado este autor (MARTÍN CRIADO, 2004, p. 96), enfocándose en los usos que Bourdieu hace de él en Homo academicus y en La nobleza de Estado. Señala que este tipo de análisis presenta, entre otras ventajas, la de permitir distinguir entre dinámicas de génesis y de mantenimiento de las instituciones y sus efectos. Y explica que históricamente se han conformado ámbitos de relaciones sociales 
que con el tiempo se han autonomizado de los poderes que les dieron origen; pero además, las luchas entre posiciones -definitorias del campo en cuestión- implican alianzas de los agentes con poderes exteriores al campo, con lo que la autonomía del campo es, tal como exhibe Bourdieu, siempre relativa. Esto le ha permitido al autor de Homo academicus analizar el campo universitario como un espacio de constantes luchas (de los profesores por conservar o mejorar sus posiciones, en el marco de una serie de transformaciones sociales más o menos externas al campo, que sin embargo interfieren en él), todo lo cual le posibilita visualizar la transformación de la estructura del campo y la modificación, entre otros aspectos, de las carreras docentes o las jerarquías entre disciplinas. Sin embargo, para Martín Criado el libro no logra deshacerse por completo del esquema funcionalista, al explicar los movimientos en la universidad en Mayo del 68 como "un estado crítico del sistema que serviría para analizar mejor el estado orgánico” (2004, p. 98).

Al pasar al análisis de La nobleza de Estado, el crítico español considera que el texto promete un análisis en términos de campo, pero regresa continuamente al enfoque y a los temas del funcionalismo. Así, el análisis arroja que las escuelas de élite (grandes écoles) dentro del campo universitario son tanto campo de luchas como estructura cuya función es la reproducción social. En definitiva y desde su óptica, el concepto bourdieano de campo (heredero del pensamiento weberiano) promete un análisis fructífero; no obstante, cuando Bourdieu lo emplea, vuelve a caer en las trampas del funcionalismo. No es esto un mero problema retórico, sino que acarrearía problemas de método, al limitar las posibilidades del análisis histórico.

Creemos que el trabajo analítico emprendido por Martín Criado sobre la obra del sociólogo francés en el texto antecitado es minucioso y certero al visibilizar la coexistencia de dos principios explicativos

Sabido es que no se trat de la primera vez que se atribuyó a Bourdieu la cualidad de funcionalista. El catedrático españo Raimundo Cuesta (2010) nos recuerda que este tipo de crítica se remonta a principios de la década de 1970. Años después en 1985, el teórico alemán Peter Bürger calificó de funcionalistas los estudios de Bourdieu sobre el campo literario. Ello motivó una muy breve respuesta en laque el sociólogo francés consideró que además de realizar un abordaje reduccionista de su trabajo, el calificativo de Bürger podía ser considerado una suerte de insulto académico imposible de contrastar (BOURDIEU, 1986/1990). contrapuestos, uno de ellos funcionalista. ${ }^{8}$ Efectivamente, las argumentaciones funcionalistas están presentes en numerosas ocasiones en sus textos. Sin embargo, no consideramos que esto haya tenido una fuerza obturadora tal que el énfasis teórico puesto en los mecanismos de reproducción le haya impedido a Bourdieu visibilizar los cambios sociales o la dimensión histórica a lo largo de sus investigaciones. Muy lejos de ello, sus análisis en términos de campos proponen precisamente lo contrario: abren la mirada al juego de las luchas sociales y a las transformaciones estructurales de los campos y del espacio social a cada momento. La presencia de “contradicciones" o de principios teóricos y lógicas contrapuestas en las explicaciones de las prácticas sociales es, en alguna medida, parte inexpugnable del trabajo productivo de cualquier sociólogo. Todo análisis es perfectible. Es labor de la crítica, entonces, ponderar la validez de las elaboraciones teóricas con las que hasta aquí contamos y su potencialidad para continuar impulsando una 
pesquisa fructífera. Si la explicación funcionalista huelga, consideramos que simplemente podemos prescindir de ella en un futuro programa de investigaciones en el campo educativo.

En cambio, los aportes bourdieanos en lo que hace a la teoría de los campos y del espacio social son su mejor legado. Se trata, no obstante, de un arsenal que ha sido abordado también desde lecturas críticas, entre ellas y como adelantáramos páginas atrás, la del sociólogo francés Bernard Lahire (2005). Nos detendremos en ello a continuación.

\section{SOBRE LOS CONCEPTOS DE CAMPO Y HABITUS}

Lahire (2005) señala acertadamente que, como todo buen concepto sociológico, el concepto de campo aumenta la imaginación científica y conduce a realizar tareas empíricas inéditas, que quizás jamás se hubieran emprendido de no haber contado con esa herramienta. No obstante, la teoría de los campos está lejos de explicar la totalidad de las prácticas sociales, pues presenta lagunas y obstáculos que es necesario identificar. En primer término, cuestiona la noción de autonomía de los campos, ya que puede conducir a una ilusión de separación completa de actividades que, aunque válida para determinados niveles de análisis, no lo sería tanto para otros. Se da el caso de que campos como el económico -con el mercado como rector- el político o el jurídico, atraviesan y "cubren" la totalidad de la vida social, configurándola de variadas maneras. Con esto deja planteada la cuestión de que sería mejor hablar de "lógicas, funciones o dimensiones de la vida social, más que universos o esferas realmente separadas" (LAHIRE, 2005, p. 39). ${ }^{9}$

Así, la reducción de todos los fenómenos al marco conceptual de los campos relativamente autónomos constituye, para este autor, una generalización abusiva. La teoría de los campos es más bien una "teoría regional" antes que una teoría general que pueda explicar todas las prácticas. ${ }^{10}$ Por otro lado, ciertas actividades no forman parte de ningún campo, no pueden ser inscriptas dentro de esta lógica. Están, al decir de Lahire (2005), “fuera de campo”, y por ende, no son objeto del análisis sociológico en la teoría bourdieana. Esta situación involucra a gran parte de los individuos de nuestra sociedad, que si bien sí integran lo que Bourdieu denomina el espacio social (organizado según el volumen y la estructura del capital poseído), sus prácticas están fuera de campo al quedar excluidas del campo de poder (como en el caso particular de las clases menos favorecidas). Es el caso también de aquellas actividades de las que participamos de manera temporal porque "no están organizadas sistemáticamente en forma de espacios de posiciones de lucha entre diferentes agentes que ocupan tales posiciones" (LAHIRE, 2005, p. 42). Actividades de la vida cotidiana, tareas efímeras o poco significativas en términos de lucha por el poder quedan así fuera del análisis. La teoría de

9 Es interesante señalar cómo vuelve a aparecer aquí la terminología funcionalista y ello no genera ningún tipo de inconveniente para Lahire. 10 La crítica refiere, a modo de ejemplo en este punto, a lo que Bourdieu ha denominado "campo familiar". Lahire (2005) señala la inconveniencia de conceptualizar a la familia de ese modo, pues las actividades que en ella se realizan distan mucho de compartir la misma lógica que está presente en otros campos, como el artístico o el literario. por tomar otros casos. En éstos se puede participar como profesional o como aficionado, como productor y miembro permanente, o como consumidor y miembro ocasional, resalta. En una familia, en cambio, las actividades de padre, madre o cónyuge no se "practican", no se ofrecen como espectáculo, no hay miembros permanentes y otros aficionados. De manera que si se la compara con el campo artístico, la familia nada tiene en común y dista mucho de poder ser considerada un campo con idéntico modo de funcionamiento (LAHIRE, 2005). La familia como campo sería uno de esos ejemplos de generalización abusiva en donde ha sido (mal) aplicado el concepto de campo y en esto coincidimos con el crítico. 
los campos puede constituirse entonces en un obstáculo para el estudio de este tipo de fenómenos, pues caen fuera de ella.

En el caso de la escuela, eventos como cierto tipo de experiencias subjetivas, vivencias y emociones de quienes transitan por las aulas pueden quedar fuera de campo, desde esta óptica. Efectivamente, otros marcos teóricos proveen elementos para su indagación que la perspectiva de los campos excluye. ${ }^{11}$ Es decir, Bourdieu jamás ignora que los sujetos elaboran representaciones y significados, pero su teoría aporta herramientas insuficientes para el análisis. La ausencia de un método particular para el abordaje de los discursos conduce a una lectura desprovista de las herramientas del sociólogo, lo que implica abordarlos "sin otra cosa que su sensatez de lectores profesionales por todo equipaje” al decir de Lahire (2005, p. 57).

Otro aspecto de las prácticas escolares que no es indagado por la teoría bourdieana es el carácter específico de los saberes que ella transmite. Nos deja En la escuela de Dubet y Martuccelli (1998), por ejemplo, desarrolla una sociología de la experiencia escolar en la que no sólo las estrategias de los/as estudiantes, sino también los procesos de socialización y subjetivación que experimentan a lo largo de sus trayectorias académicas son objeto de análisis. Por otra parte, la consideración de que las políticas educativas no son otra cosa que políticas de subjetividad tiene plena vigencia en los debates actuales (TEDESCO, 2012)

12

El concepto de habitus ha resultado objeto de diversas recepciones, usos y críticas.

Para el caso de Estados

Unidos, cabe resaltar aquellas "interpretaciones

que lo consideran una fuente ilimitada de creatividad de los agentes hasta otras que lo ven como la máscara de una visión mecánica de la reproducción y el dominio" (WACQUANT apud MARTINEZ, 2008, p. 265).

13

De la existencia de un desajuste entre habitus y situaciones, así como de la falta de coherencia de las acciones socializadoras también se ha ocupado Criado (2010).
[...] inermes en lo tocante a la especificidad de la escuela, a saber, los contenidos (históricamente variables) de las actividades que se traman y de los saberes que en ella circulan, los gestos de estudio que allí se transmiten, las disposiciones que se constituyen y reconstituyen incesantemente, las formas de relaciones pedagógicas (que son también relaciones de poder) que se entablan, etc. (LAHIRE, 2005, p. 55-56)

En definitiva, es dable identificar situaciones, significados y prácticas que quedan fuera de estudio. Ello no invalida la teoría bourdieana del sistema de enseñanza en su conjunto -no es ésa la pretensión de Bernard Lahire- sino que invita a continuar explorando otros marcos categoriales que puedan resultar útiles para la comprensión de fenómenos altamente complejos como los que tienen lugar en las instituciones educativas contemporáneas

Un segundo concepto sobre el que Lahire ha focalizado la crítica de modo fructífero es la noción de habitus. Recordemos que para Bourdieu se trata de un sistema de disposiciones a la acción, percepción y reflexión que se constituye durante la socialización de los sujetos (en la infancia, fundamentalmente) y resulta duradero y transferible a diversas situaciones. ${ }^{12}$ El primer problema de esta definición, señala Lahire, es la idea de "sistema", pues supone una cierta homogeneidad del conjunto de disposiciones, que las liga y articula, y permite su puesta en práctica ante escenarios diferentes. Tal homogeneidad dentro de dicho sistema hablaría de una cierta similitud de experiencias socializadoras. ${ }^{13}$ 


\begin{abstract}
Yo sostengo que al observar el mundo social uno se da cuenta de que existen un millón de experiencias socializadoras que son heterogéneas y que, incluso, pueden ser contradictorias entre sí. Esto quiere decir que no tenemos incorporado un sistema coherente y homogéneo de disposiciones que puede transferirse para todos lados, sino que somos el producto de disposiciones heterogéneas y muchas veces contradictorias. [...] No veo un sistema de disposiciones homogéneo sino un patrimonio de disposiciones que sólo son homogéneas si la socialización fue homogénea, pero que la mayoría de las veces es heterogénea. Incluso en el interior de una familia hay heterogeneidad. (LAHIRE 2008 apud GESSAGHI; SENDÓN, 2008, p. 72)
\end{abstract}

Dada una heterogeneidad de disposiciones que se encuentra en el origen de las prácticas, no será posible entonces hablar de la durabilidad y posibilidad de transferencia de un único habitus, como sistema unificado y coherente, para el autor. He aquí el problema de la transferibilidad del habitus: no son las mismas disposiciones las que se transfieren de una situación a otra, sino que en cada situación, los agentes ponen en juego disposiciones diferentes. De acuerdo a la situación de que se trate, se activarán determinadas disposiciones, mientras que otras quedarán inhibidas, a lo que Lahire se refiere como el "hombre plural". Desde esta perspectiva, los agentes siguen produciendo prácticas y discursos adaptados a la situación las más de las veces, y en esto no difieren el crítico y la perspectiva bourdieana.

En síntesis, Lahire invita a emplear de modo heterodoxo y no dogmático la teoría de Pierre Bourdieu, proponiendo reelaboraciones necesarias y sacando provecho de su potencial.

\title{
SOBRE EL CONCEPTO DE CAMPO EN LA PERIFERIA: RECAUDOS TEÓRICO-METODOLÓGICOS
}

Autora de una serie de investigaciones que han hecho un uso fructífero de la teoría bourdieana en Argentina, Ana Teresa Martínez ha escrito, retomando palabras del propio autor, lo siguiente:

\footnotetext{
Al proponer de manera práctica en sus investigaciones una manera de estudiar el mundo social en términos de campo, Bourdieu ofreció algunos "primeros resultados, provisorios y revisables" e "indicó una dirección" para un "programa de investigaciones empíricas realmente integradas y acumulativas". Con esto, pretendía proponer una manera diferente (no fisicalista ni empirista) de realizar la "ambición legítima de sistematicidad que encierran las pretensiones totalizantes de la "gran teoría". (MARTINEZ, 2007, p. 2-3. Comillas en el original)
} 
Resaltamos la vocación integradora y acumulativa presente en la investigación sociológica existente y su necesidad a futuro. Es una casi una obviedad afirmar que la sociología no podría darse el lujo de empezar de cero cada vez, obviedad que sin embargo parece preciso traer a colación. Esa es otra de las varias razones que esgrimimos en este artículo para procurar dar continuidad al programa de pesquisa bourdieano. Como hemos ya expresado, eso no implica tomar en bloque todo su esquema teórico ni mucho menos, pretender arribar a los mismos resultados en escenarios sociales diferentes. En todo caso, la tarea pendiente puede ser, con las reelaboraciones teóricas que correspondan, arribar a resultados comparables con aquellos a los que el sociólogo francés arribó.

Del fragmento antecitado de Martinez nos interesa, además, resaltar la importancia de la manera bourdieana de mirar el mundo social, alejada del fisicalismo o empirismo. En la misma dirección, Alicia Gutiérrez (2011) nos alerta sobre las modalidades esencialistas de lectura de las investigaciones de Bourdieu, reafirmando el carácter relacional de las prácticas en cualquier campo. Estos recaudos resultan particularmente válidos para el estudio del campo escolar, en donde las explicaciones funcionalistas brindadas por el propio autor podrían convertirse en esquemas "encorsetantes" para futuros programas investigativos. Si la escuela contribuye o no a reproducir la estructura del espacio social y, más importante aún, si es posible que desde la acción escolar esa relación se modifique, es algo que deberá establecerse en cada caso.

El trabajo de Ana Teresa Martinez (2007) sobre el estudio de campos periféricos nos permite extraer, por otra parte, algunas conclusiones o postulados teórico-metodológicos. En primer término, el carácter periférico de los campos que dan forma a los espacios sociales latinoamericanos puede apreciarse a partir del análisis de sus articulaciones con otros campos locales, así como con los de las sociedades centrales. En segundo término, no debe soslayarse el carácter productivo e inventivo, al igual que las especificidades que los campos periféricos y los espacios sociales locales presentan. Por último, el carácter nacionalmente más homogéneo o integrado de las sociedades estudiadas por Bourdieu en Francia y Argelia en nada impide que la teoría de los campos pueda emplearse en espacios sociales más fragmentados en términos nacionales. Las sociedades latinoamericanas somos producto de la colonización y nuestros estados "nacionales" no necesariamente abarcan en su seno a poblaciones de un mismo origen nacional. Martinez pone en evidencia, a través de su propia pesquisa, que ello no es un obstáculo a la hora de poner en acción el arsenal bourdieano en nuestras sociedades periféricas, que en mucho se diferencian -no tendría por qué ser de otro modo- de las centrales. 


\section{COMENTARIOS FINALES. LA ESCUELA Y SUS POSIBILIDADES DE CONTRIBUIR A LA DEMOCRATIZACIÓN SOCIAL}

El interjuego de las estrategias familiares de reproducción social y de la acción del sistema de enseñanza contribuye a la reproducción de la estructura social, según Pierre Bourdieu. Desde su perspectiva, resulta escasamente eficaz la acción que la escuela podría producir con vistas a transformar drásticamente la estructura de distribución del capital en el espacio social y beneficiar así a las clases más desfavorecidas.

El sistema de enseñanza puede, como ocurrió a lo largo del siglo XX en la mayoría de los países del mundo occidental, volverse masivo. El capital cultural "distribuido" por la escuela alcanza entonces y en mayor medida que décadas atrás, a estudiantes de orígenes sociales más bajos. Sus recorridos escolares presentan en las últimas décadas mayor duración y muchos de ellos han alcanzado niveles educativos que la generación anterior no alcanzó. Sin embargo, los títulos escolares y los conocimientos adquiridos han revelado tener un valor relativo, que se pone en juego en el mercado de bienes simbólicos. Ha tenido lugar una devaluación de las credenciales educativas a medida que la escolaridad se ha ido masificando. El título académico ha perdido así parte del poder de otorgar jerarquía a su titular que antaño tenía. Paralelamente, el sistema educativo ha experimentado un continuo proceso de fragmentación (TIRAMONTI, 2005). A distintos establecimientos asisten alumnos/as de origen social también diverso, razón por la cual adquiere una importancia simbólica progresiva (que se retraduce en beneficios económicos concretos en el mercado laboral) el itinerario escolar realizado por cada grupo de agentes y el tipo de establecimientos por los que ha transitado. Aunque no se trate nunca de predicciones absolutas, los recorridos escolares permiten aventurar ciertas posibilidades de éxito en los estudios superiores, oportunidades de acceso al mercado de trabajo o, incluso, determinadas expectativas de futuro de parte de los propios estudiantes.

Durante los últimos años antes de su muerte, ocurrida en 2002, Bourdieu hizo un esfuerzo por formular aquellas consecuencias de sus investigaciones que pudieran resultar eficientes para intervenciones pedagógico-políticas transformadoras de esa sociedad tan injusta y desigual que aparece descrita en sus obras. Este esfuerzo deriva, quizás, de las críticas recibidas, motivadas algunas de ellas por la aparente imposibilidad de burlar los mecanismos de la reproducción que se desprende de sus textos. "Es porque conocemos las leyes de la reproducción por lo que tenemos alguna oportunidad de minimizar la acción reproductora de la institución escolar" ha expresado (BOURDIEU, 2005, p. 160).

Una transformación necesaria está pendiente aún en el plano simbólico-discursivo. El sistema escolar puede y debe dejar de presentarse 
a sí mismo como la institución capaz de asegurar la democratización social a través de la distribución equitativa de la cultura a todos los estudiantes y reconocer que, en cambio, si algo puede hacer es, en principio, no reforzar más las desigualdades preexistentes a través del discurso escolar.

\begin{abstract}
Si yo fuera ministro, la primera recomendación que haría a los profesores sería: no hacer jamás juicios de valor sobre sus alumnos; ustedes no tienen derecho a emplear la palabra "idiota", ustedes no tienen derecho a emplear la palabra "estúpido" [...] deberían saber y comprender que tienen un poder diabólico de nominación, de constitución que se ejerce sobre la identidad misma de los adolescentes, sobre su imagen de sí y que pueden infligir traumatismos terribles. (BOURDIEU, 2005, p. 161)
\end{abstract}

De este modo, recalca la necesidad de desarmar el discurso de la escuela como institución esencialmente meritocrática y garante de una supuesta igualdad de oportunidades, institución que en los hechos termina ocultando, y con ello, naturalizando diferencias que, como sabemos, son sociales. Un gran paso adelante se daría si se comienza por evitar reforzar los privilegios, se los visibiliza -a través de la investigación en el campo educativo, por ejemplo- y se activan posibles mecanismos de compensación.

\footnotetext{
Para combatir lo que yo llamo efecto de destino, y la tendencia de los profesores a creer que ellos juzgan a personas cuando en verdad juzgan a individuos sociales, habría que darles la conciencia de los mecanismos más elementales de la transmisión cultural. Esto sería importante para sensibilizar a los profesores y al menos poner en duda sus creencias. (BOURDIEU, 2005, p. 174)
}

Con esta afirmación, defiende al mismo tiempo la necesidad de ofrecer a todos los profesores, de todas las disciplinas, herramientas de la sociología de la educación e historia de las ciencias, para que tengan conciencia de qué es lo que hacen cuando enseñan y evalúan a sus alumnos en las aulas, o qué efectos sociales tienen sus acciones pedagógicas.

En definitiva, nos parece fundamental recuperar el legado bourdieano, tanto en sus conclusiones más graves y desesperanzadas referidas a la reproducción social resultante de la acción escolar, como en la apertura al juego de las luchas y resistencias sociales con la idea de campo escolar que guía sus obras de madurez.Las primeras investigaciones nos han posibilitado a los sociólogos, incluso a quienes habitamos en sociedades periféricas, elaborar recaudos teórico-metodológicos que 
deberán servirnos en indagaciones futuras. Las obras de los últimos años, en tanto, nos invitan a extraer conclusiones y diseñar políticas y pedagogías para una escuela que no contribuya a reproducir el mundo $\mathrm{y}$, en consecuencia, colabore a democratizarlo.

\section{REFERENCIAS}

BAUMAN, Zygmunt. Sobre la educación en un mundo líquido. Conversaciones con Ricardo Mazzeo. Buenos Aires: Paidós, 2013.

BOURDIEU, Pierre. Cosas dichas. Buenos Aires: Gedisa, 1988.

BOURDIEU, Pierre. The intellectual field: a world apart. En: BOURDIEU, Pierre. In other words: essays towards a reflexive sociology. California: Stanford University Press, 1990. p. 140-149. Trabajo original publicado en 1986.

BOURDIEU, Pierre. Capital cultural, escuela y espacio social. Buenos Aires: Siglo XXI, 2005.

BOURDIEU, Pierre. La distinción. Criterio y bases sociales del gusto. 3ra. ed. Madrid: Taurus, 2006.

BOURDIEU, Pierre. Homo academicus. Buenos Aires: Siglo XXI, 2008. Trabajo original publicado en 1984.

BOURDIEU, Pierre. Las estrategias de la reproducción social. Buenos Aires: Siglo XXI, 2011.

BOURDIEU, Pierre. La nobleza de Estado: Educación de elite y espíritu de cuerpo. Buenos Aires: Siglo XXI, 2013. Trabajo original publicado en 1989.

BOURDIEU, Pierre; PASSERON, Jean-Claude. La reproducción. Elementos para una teoría del sistema de enseñanza. México: Fontamara, 1996. Trabajo original publicado en 1970.

BOURDIEU, Pierre; PASSERON, Jean-Claude. Los herederos. Los estudiantes y la cultura. Buenos Aires: Siglo XXI, 2006. Trabajo original publicado en 1964.

CUESTA, Raimundo. Reseña de Martín Criado, E. La escuela sin funciones. Crítica de la sociología de la educación crítica. Reseñas educativas, Barcelona, n. 13, 3 nov. 2010. Disponible en <http://edrev.asu.edu/edrev/index.php/ER/article/viewFile/1528/195>. Acceso: 10 mayo 2016.

DUBET, François. ¿Por qué preferimos la desigualdad? (aunque digamos lo contrario). Buenos Aires: Siglo XXI, 2015.

DUBET, François; MARTUCCELLI, Danilo. En la escuela. Sociología de la experiencia escolar. Buenos Aires: Losada, 1998.

GARCÍA CANCLINI, Néstor. Introducción: la sociología de la cultura de Pierre Bourdieu. En: BOURDIEU, P. Sociología y cultura. México: Grijalbo, 1990.

GESSAGHI, Victoria; SENDÓN, María Alejandra. Socializaciones y disposiciones heterogéneas: sus vínculos con la escolarización. Entrevista a Bernard Lahire. Propuesta Educativa, Buenos Aires, n. 30, p. 71-77, 2008. Disponible en: <http://www.propuestaeducativa.flacso.org.ar/ archivos/entrevistas/30.pdf>. Acceso: 22 ago. 2013.

GIROUX, Henry A. Pedagogía y política de la esperanza. Teoría, cultura y enseñanza. Buenos Aires: Amorrortu, 2004.

GUTIÉRREZ, Alicia. Clases, espacio social y estrategias. En: BOURDIEU, P. Las estrategias de la reproducción social. Buenos Aires: Siglo XXI, 2011.

KAPLAN, Carina Viviana. Talentos, dones e inteligencias: el fracaso escolar no es un destino. Buenos Aires: Colihue, 2008.

LAHIRE, Bernard. Campo, fuera de campo, contracampo. En: LAHIRE, B. (Dir.) El trabajo sociológico de Pierre Bourdieu: deudas y críticas. Buenos Aires: Siglo XXI, 2005. p. 29-69.

MARTÍN CRIADO, Enrique. Una crítica de la sociología de la educación crítica. Anduli. Revista Andaluza de Ciencias Sociales, n. 2, p. 9-27, 2003. 
MARTÍN CRIADO, Enrique. De la reproducción al campo escolar. En: ALONSO, L. E.; MARTÍN CRIADO, E.; MORENO PESTAÑA, J. L. (Eds.) Pierre Bourdieu: las herramientas del sociólogo. Madrid: Fundamentos, 2004. p. 67-114.

MARTÍN CRIADO, Enrique. La escuela sin funciones. Crítica de la sociología de la educación crítica. Barcelona: Bellaterra, 2010.

MARTINEZ, Ana Teresa. Para estudiar campos periféricos. Un ensayo sobre las condiciones de utilización fecunda de la teoría del campo de Pierre Bourdieu. Trabajo y sociedad: indagaciones sobre el trabajo, la cultura y las prácticas políticas en sociedades segmentadas, Santiago del Estero, Argentina, v. 9, n. 9, 31 p., invierno 2007. Disponible en: <http://www.unse.edu.ar/ trabajoysociedad/Martinez.pdf>. Acceso: 10 mayo 2016.

MARTINEZ, Ana Teresa. Lectura y lectores de Pierre Bourdieu. Lecciones y equívocos desde Francia y EEUU. Cuadernos de la Facultad de Humanidades y Ciencias Sociales, Jujuy, n. 34, p. 251268, mayo 2008. Disponible en: <http://www.redalyc.org/articulo.oa?id=18519814017>. Acceso: 12 mayo 2016.

MORAÑA, Mabel. Bourdieu en la periferia: capital simbólico y campo cultural en América Latina. Santiago de Chile: Cuarto Propio, 2014.

SEGATO, Rita. La crítica de la colonialidad en ocho ensayos y una antropología por demanda. Buenos Aires: Prometeo, 2015.

TEDESCO, Juan Carlos. Educación y justicia social en América Latina. Buenos Aires: FCE/ Universidad Nacional de San Martín, 2012.

TIRAMONTI, Guillermina. La fragmentación educativa y los cambios en los factores de estratificación. En: TIRAMONTI, G. (Comp.). La trama de la desigualdad educativa: mutaciones recientes en la escuela media. Buenos Aires: Manantial, 2005. p. 15-46.

TORRADO, Susana. Historia de la familia en la Argentina moderna (1870-2000). Buenos Aires: Ediciones de la Flor, 2003.

\section{MERCEDES MOLINA GALARZA}

Consejo Nacional de Investigaciones Científicas y Técnicas -CONICET-, Argentina; Universidad Nacional de Cuyo, Mendoza, Argentina mmolina@mendoza-conicet.gob.ar 